Simulation of plasma turbulence in scrape-off layer conditions: the GBS code, simulation results and code validation

This article has been downloaded from IOPscience. Please scroll down to see the full text article.

2012 Plasma Phys. Control. Fusion 54124047

(http://iopscience.iop.org/0741-3335/54/12/124047)

View the table of contents for this issue, or go to the journal homepage for more

Download details:

IP Address: 128.178.125.98

The article was downloaded on 23/11/2012 at 08:21

Please note that terms and conditions apply. 


\title{
Simulation of plasma turbulence in scrape-off layer conditions: the GBS code, simulation results and code validation
}

\author{
P Ricci, F D Halpern, S Jolliet, J Loizu, A Mosetto, A Fasoli, I Furno and \\ C Theiler \\ Ecole Polytechnique Fédérale de Lausanne (EPFL), Centre de Recherches en Physique des Plasmas \\ Association Euratom-Confédération Suisse, CH-1015 Lausanne, Switzerland \\ E-mail: paolo.ricci@epfl.ch
}

Received 3 July 2012, in final form 10 October 2012

Published 21 November 2012

Online at stacks.iop.org/PPCF/54/124047

\begin{abstract}
Based on the drift-reduced Braginskii equations, the Global Braginskii Solver, GBS, is able to model the scrape-off layer (SOL) plasma turbulence in terms of the interplay between the plasma outflow from the tokamak core, the turbulent transport, and the losses at the vessel. Model equations, the GBS numerical algorithm, and GBS simulation results are described. GBS has been first developed to model turbulence in basic plasma physics devices, such as linear and simple magnetized toroidal devices, which contain some of the main elements of SOL turbulence in a simplified setting. In this paper we summarize the findings obtained from the simulation carried out in these configurations and we report the first simulations of SOL turbulence. We also discuss the validation project that has been carried out together with the GBS development.
\end{abstract}

(Some figures may appear in colour only in the online journal)

\section{Introduction}

Turbulence in the scrape-off layer (SOL) of magnetic confinement devices is an outstanding open issue in magnetic fusion [1]. In this region, plasma interacts with the solid wall of the device, determining the boundary conditions for the core plasma, and controlling the plasma refueling, heat losses, and impurity dynamics, largely governing the fusion power output of the entire device. Persisting uncertainties related to SOL transport continue to undermine our ability to reliably predict the performance of future fusion reactors such as ITER [2].

In the SOL region, a wide range of spatiotemporal scales is involved and a number of approximations that are typically used in the study of the core plasma (e.g. small-amplitude fluctuations and the separation of turbulent and equilibrium scale lengths) are not valid. The analysis is complicated by a magnetic geometry that is particularly complex, due to the presence of open field lines and an x-point geometry. The origin of a high-confinement regime, where turbulence is suppressed and the formation of a plasma pedestal is observed, is still under debate: it is commonly believed that turbulent nonlinear phenomena, partially occurring in the SOL, play a fundamental role in suppressing the plasma transport. A full nonlinear treatment of the plasma dynamics is therefore necessary $[3,4]$.

At the edge of a tokamak, the low plasma temperature makes the collision rate large, trapped particle effects weak, and the attainment of a local thermodynamic equilibrium quick. A few moments of the particle distribution functions (i.e. the density, momentum and temperature) can be used to model the dynamics of turbulence in this region. The fluidlike equations that describe collisional plasma turbulence in the edge were summarized by Braginskii in 1965 [5]. Later, a number of reduced models more suited for computational treatment were deduced [6-10], in some cases taking into account finite Larmor radius (FLR) effects and introducing kinetic effects neglected in the original Braginskii equations. A number of codes have then been developed based on the reduced models (see, e.g. [11-15]).

In past years, focusing mostly on the closed flux surface region, the study of edge turbulence has been addressed mainly in three-dimensional flux-tube geometry, which considers 
a volume that follows the magnetic field lines and has a limited extension in the directions perpendicular to it (see, e.g. [16,17]). The turbulence drive (i.e. the density and temperature gradients) is typically fixed and imposed a priori as a background plasma profile, according to experimental measurements, and the dynamics of smallamplitude turbulence is followed. Within this approach, the regimes of edge turbulence have been identified as a competition between drift waves, resistive ballooning, and ideal MHD modes [18, 19].

On the other hand, the formation of a self-consistent equilibrium in the SOL region-as a balance between the plasma source from the tokamak core, turbulent transport, and field-line end losses at the limiter or divertor plates - has been studied within a two-dimensional approximation [20-22]. It has been found that the dynamics in this region is primarily characterized by the interchange mode and by the sheathdriven mode. The turbulence has an amplitude comparable to the background plasma and can be bursty, with intermittent cross-field transport events, the so-called plasma blobs. The SOL dynamics has been coupled to the closed flux surface region modeled with a Hasegawa-Wakatani two-dimensional model [23, 24].

In general, little work has been performed on global three-dimensional simulations that evolve plasma profiles and turbulence self consistently. The obtained results suggest that an increase of the plasma source strength can lead to a higher plasma confinement, associated with the stabilization of the resistive X-point mode in divertor geometry [25, 26]. Simulations also show that ELMs are possibly associated with the nonlinear evolution of peeling ballooning modes [27-29].

In the last few years, the Global Braginskii Solver code, GBS, has been developed with the goal of simulating plasma SOL turbulence by evolving the full profiles of the various quantities with no separation between 'perturbations' and 'equilibrium'. These simulations can explore the selfconsistent evolution and structure of the plasma profiles in the presence of (i) plasma density and heat input from the core of the fusion machine, (ii) cross-field transport produced by plasma instabilities (interchange instability or drift waves, for example), and (iii) parallel losses at the sheaths where the magnetic field lines terminate on the walls.

In order to progressively approach the complexity of tokamak edge simulations, the GBS code was initially developed and used to simulate turbulent dynamics in basic plasma physics devices of increasing complexity [30-39]. Containing some of the main elements of SOL plasma dynamics, some of these devices offer a simple and welldiagnosed testbed to study the basic physics of plasma edge turbulence and the associated transport of heat and particles in a simplified setting. In particular, the initial GBS simulations were focused on linear devices, such as LAPD [40], and on the simple magnetized plasma configuration (SMT), such as the TORPEX device $[41,42]$, in which a vertical magnetic field, superimposed on a toroidal field, creates helicoidal field lines with both ends terminating on the torus vessel. The first version of the code was able to follow two-dimensional plasma dynamics in the plane perpendicular to the magnetic field [30$32,34,37,39]$, and was developed from ESEL [13,14], a code that implements the algorithm described in [15]. The two-dimensional code was used for the simulation of idealinterchange SMT turbulence, based on the fact that in this regime $k_{\|}=0$. GBS was then extended to the third dimension, in order to describe the dynamics in the direction parallel to the magnetic field, first to perform flux-tube simulations [33], reaching then the capability of performing global simulation of SMT and linear devices [35-38]. As described in this paper, GBS now allows simulations of the tokamak SOL.

Within the GBS development, a validation project of the GBS data has been carried out [32,37]. Validation of edge turbulence codes is challenging because of the difficult diagnostic access to tokamak plasmas and the complexity of the interpretation of the experimental measurements. Previous comparisons between experimental and simulation data from Alcator C-Mod, TCV, JET and NSTX [43-47] show partial agreement. Being applied to a well-diagnosed basic plasma physics experiment such as TORPEX, we have been able to compare the GBS results to experimental data in great detail. We have also established a rigorous framework to compare quantitatively simulations and experiments.

The goal of this paper is to present a detailed derivation of the model considered by GBS, the description of the numerical algorithm used, and of its implementation. We then summarize the results of GBS simulations of linear devices and SMT and we present the first results of SOL simulations. We also discuss the main results of the validation project that has been carried out within the GBS development.

We finally remark that more refined kinetic codes are being developed to approach the simulation of edge turbulent phenomena $[48,49]$. Even if GBS does not take into account the kinetic details of edge physics, it contains the main physics elements that presumably underlie the most important phenomena that occur in this region. It therefore provides an agile tool to explore the operating phase space of fusion devices and, because of the relative simplicity of the fluid model with respect to the kinetic one, it facilitates the theoretical understanding of simulation results.

This paper is structured as follows. After the introduction, in section 2 we report the model that is considered by GBS to simulate turbulence in SOL-relevant conditions. The numerics used in GBS is described in section 3. Section 4 is focused on the simulation results focusing on linear devices, the SMT configuration, and the tokamak SOL. We describe the code validation methodology and an example of GBS validation in section 5. The conclusions and the outlook follow.

\section{The plasma turbulence model}

At the plasma edge, where collisionality plays a dominant role and kinetic effects such as particle trapping and waveparticle resonance are less important, fluid modeling is still an appropriate choice in order to perform global turbulence simulations at a computational cost that allows a wide parameter scan. Within the hypotheses that (i) the plasma distribution function is close to a local Maxwellian, (ii) the macroscopic time variations are slower than the collisional time, (iii) the scale lengths along the magnetic field are longer 
than the mean free path, and (iv) the perpendicular scale lengths are longer than the ion gyroradius, the motion of the plasma can be described by the Braginskii momentum equation [5], which for the species $\alpha=\{\mathrm{i}, \mathrm{e}\}$, state that

$$
\begin{aligned}
& m_{\alpha} n\left(\frac{\partial}{\partial t}+\boldsymbol{v}_{\alpha} \cdot \nabla\right) \boldsymbol{v}_{\alpha}=-\nabla p_{\alpha} \\
& \quad+q_{\alpha} n\left(\boldsymbol{E}+\frac{1}{c} \boldsymbol{v}_{\perp \alpha} \times \boldsymbol{B}\right)-\nabla \cdot \underline{\underline{\pi_{\alpha}}}+\boldsymbol{R}_{\alpha}
\end{aligned}
$$

where we have assumed a quasi-neutral plasma with singly charged ions, such that $n=n_{\mathrm{i}}=n_{\mathrm{e}}$ and $q_{\mathrm{i}}=-q_{\mathrm{e}}=e$. The stress tensor is divided into a FLR part and a viscous part, $\underline{\underline{\pi_{\alpha}}}=\underline{\underline{\pi_{\alpha}}}{ }^{\text {FLR }}+\underline{\underline{\pi_{\alpha}}}$ vis , such that

$$
\begin{aligned}
& \nabla \cdot{\stackrel{\underline{\pi_{\alpha}}}{ }}^{\mathrm{FLR}}=-m_{\alpha} n \boldsymbol{v}_{d \alpha} \cdot \nabla \boldsymbol{v}_{\alpha}+p_{\alpha}\left(\nabla \times \frac{\boldsymbol{b}}{\omega_{c \alpha}}\right) \cdot \nabla \boldsymbol{v}_{\alpha} \\
& \quad+\nabla_{\perp}\left[\frac{p_{\alpha}}{2 \omega_{c \alpha}} \nabla \cdot\left(\boldsymbol{b} \times \boldsymbol{v}_{\alpha}\right)\right]+\boldsymbol{b} \times \nabla\left(\frac{p_{\alpha}}{2 \omega_{c \alpha}} \nabla_{\perp} \cdot \boldsymbol{v}_{\alpha}\right)
\end{aligned}
$$

and

$$
\boldsymbol{\nabla} \cdot{\underline{\underline{\pi_{\alpha}}}}^{\text {vis }}=G_{\alpha} \boldsymbol{\kappa}-\frac{1}{3} \nabla G_{\alpha}+\left[(\boldsymbol{b} \cdot \nabla) G_{\alpha}\right] \boldsymbol{b}+G_{\alpha}(\boldsymbol{\nabla} \cdot \boldsymbol{b})
$$

having defined the diamagnetic drift-velocity, $\boldsymbol{v}_{d \alpha}=c \boldsymbol{b} \times$ $\nabla p_{\alpha} /\left(q_{\alpha} n B\right)$, the field-line curvature $\boldsymbol{\kappa}=\boldsymbol{b} \cdot \boldsymbol{\nabla} \boldsymbol{b}$, the stress function $G_{\alpha}=-3 \eta_{0 \alpha}\left[\nabla_{\|} V_{\| \alpha} \boldsymbol{b}-\boldsymbol{\kappa} \cdot \boldsymbol{v}-\boldsymbol{\nabla} \cdot \boldsymbol{V}_{\alpha} / 3\right], \boldsymbol{b}=\boldsymbol{B} / \boldsymbol{B}$, and the viscosities $\eta_{0 \alpha}=\xi_{\alpha} p_{\alpha} \tau_{\alpha}$, with $\tau_{\alpha}$ the collisional time for species $\alpha$ and $\xi_{\alpha}$ a coefficient that depends on the species $\left(\xi_{\mathrm{i}}=0.96, \xi_{\mathrm{e}}=0.73\right)$. The momentum transfer vector, $\boldsymbol{R}_{\alpha}$, can be neglected for ions, while it has to be kept for electrons and is equal to $\boldsymbol{R}_{\mathrm{e}}=n \boldsymbol{b}\left[e j_{\|} / \sigma_{\|}-0.71 \nabla_{\|} T_{\mathrm{e}}\right]$, with $\sigma_{\|}=1.96 n e^{2} \tau_{e} / m_{\mathrm{e}}$ being the parallel conductivity and $j_{\|}=e n\left(V_{\| \mathrm{i}}-V_{\| \mathrm{e}}\right)$. With respect to the standard Braginskii equations, the friction in the direction perpendicular to the magnetic field is neglected.

We adopt the drift ordering, which is based on assuming that $\mathrm{d} / \mathrm{d} t \ll \omega_{\mathrm{ci}}$ and that turbulence is essentially aligned with the field-line, $\left|\nabla_{\|}\right| \ll\left|\nabla_{\perp}\right|$ (see, e.g. [50] for a detailed discussion of the drift ordering). Within the drift ordering, it is useful to split the analysis of the dynamics into the direction parallel and perpendicular to the magnetic field, by decomposing $\boldsymbol{V}_{\alpha}=V_{\| \alpha} \boldsymbol{b}+\boldsymbol{v}_{\perp \alpha}$. By assuming that the viscous terms are small, the drift ordering indicates that the leading order term for $\boldsymbol{v}_{\perp}$ is $\boldsymbol{v}_{\perp \alpha 0}=\boldsymbol{v}_{d \alpha}+\boldsymbol{v}_{E}$, where $\boldsymbol{v}_{E}=c \boldsymbol{E} \times \boldsymbol{B} / B^{2}$ is the $\boldsymbol{E} \times \boldsymbol{B}$ drift. The first-order velocity is the polarization velocity, which can be expressed as

$$
\begin{aligned}
\boldsymbol{v}_{\mathrm{pol}, \alpha}= & \boldsymbol{v}_{\perp \alpha}-\boldsymbol{v}_{\perp \alpha 0} \simeq \frac{\boldsymbol{b}}{\omega_{c \alpha}} \times \frac{\mathrm{d}}{\mathrm{d} t} \boldsymbol{v}_{\perp \alpha 0} \\
& +\frac{1}{n m_{\alpha} \omega_{c \alpha}}\left\{\boldsymbol{b} \times\left[p_{\alpha}\left(\boldsymbol{\nabla} \times \frac{\boldsymbol{b}}{\omega_{c \alpha}}\right) \cdot \nabla \boldsymbol{v}_{\perp i 0}\right]\right. \\
& +\boldsymbol{b} \times \nabla_{\perp}\left[\frac{p_{\alpha}}{2 \omega_{c \mathrm{i}}} \nabla \cdot\left(\boldsymbol{b} \times \boldsymbol{v}_{\perp \alpha 0}\right)\right] \\
& \left.-\nabla_{\perp}\left[\frac{p_{\alpha}}{2 \omega_{c \alpha}} \nabla_{\perp} \cdot \boldsymbol{v}_{\perp \alpha 0}\right]\right\} \\
& +\frac{1}{n m_{\alpha} \omega_{c \alpha}} \boldsymbol{b} \times\left[G_{\alpha} \boldsymbol{\kappa}-\frac{\boldsymbol{\nabla} G_{\alpha}}{3}\right],
\end{aligned}
$$

where $\mathrm{d} / \mathrm{d} t=\partial_{t}+\left(\boldsymbol{v}_{E}+V_{\| \alpha} \boldsymbol{b}\right)$ since, from the ordering, $\boldsymbol{v}_{\mathrm{pol}}$ can be dropped and the diamagnetic drift velocity contribution is canceled out by the first term of equation (2).

Using the plasma velocity evaluated within the drift ordering, it is possible to derive the equations that are solved by GBS. Within this derivation, we consider the cold ion approximation, $T_{\mathrm{i}} \ll T_{\mathrm{e}}$, neglecting $p_{i}$ effects, except for the $G_{\mathrm{i}}$ terms (kept for numerical reasons). We remark that the cold ion approximation is fulfilled in the basic plasma physics experiments considered herein. In the SOL, and especially in the far SOL, experiments and theoretical investigations point out that turbulence is driven by the resistive ballooning mode, whose main properties are described within a cold ion model. We also assume $\boldsymbol{\nabla} \cdot \boldsymbol{b}=0$, which is generally valid in linear devices and in the SMT, while in tokamaks it corresponds to neglecting finite aspect ratio effects (tests performed with a linear stability code show that this term has a very weak impact on the SOL linear instabilities).

\subsection{Continuity equation}

For the perpendicular motion of electrons we can set $\boldsymbol{v}_{\mathrm{pol}, \mathrm{e}}=0$, since electron FLR effects are negligible and the viscous part is proportional to $m_{\mathrm{e}} / m_{\mathrm{i}}$. Therefore the electron continuity equation reads

$$
\frac{\partial n}{\partial t}+\nabla \cdot\left[n\left(\boldsymbol{v}_{E}+\boldsymbol{v}_{d \mathrm{e}}+V_{\| \mathrm{e}} \boldsymbol{b}\right)\right]=S,
$$

where $S$ is the plasma density source. Equation (5) can be rewritten as

$$
\begin{aligned}
\frac{\partial n}{\partial t}= & -\frac{c}{B}[\phi, n]+\frac{2 c}{e B}\left[n C\left(T_{\mathrm{e}}\right)+T_{\mathrm{e}} C(n)-e n C(\phi)\right] \\
& -n(\boldsymbol{b} \cdot \nabla) V_{\| \mathrm{e}}-V_{\| \mathrm{e}}(\boldsymbol{b} \cdot \nabla) n+S
\end{aligned}
$$

where the curvature operator is defined as

$$
C(A) \equiv \frac{B}{2}\left(\nabla \times \frac{b}{B}\right) \cdot \nabla A
$$

and the Poisson bracket $[A, B]$, which denotes $[A, B]=$ $\boldsymbol{b} \cdot(\nabla A \times \nabla B)$, is introduced.

\subsection{Vorticity equation}

The vorticity equation is obtained by subtracting the ion and electron continuity equations. This gives

$$
\boldsymbol{\nabla} \cdot\left(n \boldsymbol{v}_{\mathrm{pol}, \mathrm{i}}\right)+\nabla_{\|} \cdot\left(j_{\|} \boldsymbol{b}\right) / e-\nabla \cdot\left(n \boldsymbol{v}_{d \mathrm{e}}\right)=0
$$

since the ion velocity is given by $\boldsymbol{V}_{\mathrm{i}}=V_{\| \mathrm{i}} \boldsymbol{b}+\boldsymbol{v}_{E}+\boldsymbol{v}_{\mathrm{pol}, \mathrm{i}}$, having neglected the ion diamagnetic current in the cold ion limit. Equation (8) is equivalent to $\boldsymbol{\nabla} \cdot \boldsymbol{j}=0$, meaning that the displacement current is negligible and that there is no charge accumulation in the plasma.

The divergence of the polarization velocity, $\boldsymbol{\nabla} \cdot\left(n \boldsymbol{v}_{\mathrm{pol}, \mathrm{i}}\right)$, can be simplified with the hypothesis that $p_{\mathrm{i}}=0$ and using the following relation:

$$
\nabla \cdot\left(\frac{b}{B} \times\left[G_{\mathrm{i}} \kappa-\frac{\nabla G_{\mathrm{i}}}{3}\right]\right)=\frac{1}{3} \frac{\boldsymbol{b} \times \boldsymbol{\kappa}}{B} \cdot \nabla G_{\mathrm{i}} .
$$


This is derived noting that $\nabla \times(b / B) \simeq 2 b \times \kappa / 2$ and that

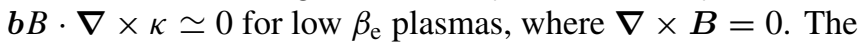
expression of the polarization current appearing in the vorticity equation can therefore be written as

$$
\boldsymbol{\nabla} \cdot n \boldsymbol{v}_{\mathrm{pol}, \mathrm{i}}=\nabla_{\perp} \cdot \frac{n c}{B \omega_{c \mathrm{i}}} \frac{\mathrm{d} \boldsymbol{E}_{\perp}}{\mathrm{d} t}+\frac{1}{3 m_{\mathrm{i}} \omega_{c \mathrm{i}}}(\boldsymbol{b} \times \boldsymbol{\kappa}) \cdot \boldsymbol{\nabla} G_{\mathrm{i}} .
$$

This expression is further simplified by using the Boussinesq approximation [51]:

$$
\nabla \cdot \frac{n c}{B \omega_{c \mathrm{i}}} \frac{\mathrm{d}}{\mathrm{d} t} \nabla_{\perp} \phi \simeq \frac{n c}{B \omega_{c \mathrm{i}}} \frac{\mathrm{d}}{\mathrm{d} t} \nabla_{\perp}^{2} \phi .
$$

This is a common approximation in the fluid community that considerably simplifies the vorticity equation. Using the fact that $\mathrm{d}_{\mathrm{i}} A / \mathrm{d} t=\partial_{t} A+c[\phi, A] / B+V_{\| \mathrm{i}}(\boldsymbol{b} \cdot \nabla) A$, the following expression is obtained:

$$
\begin{aligned}
\frac{\partial}{\partial t} \nabla_{\perp}^{2} \phi=-\frac{c}{B}\left[\phi, \nabla_{\perp}^{2} \phi\right]-V_{\| \mathrm{i}}(\boldsymbol{b} \cdot \nabla \phi) \nabla_{\perp}^{2} \phi \\
+\frac{m_{\mathrm{i}} \omega_{c \mathrm{i}}^{2}}{e}\left[(\boldsymbol{b} \cdot \nabla)\left(V_{\| \mathrm{i}}-V_{\| \mathrm{e}}\right)+\left(V_{\| \mathrm{i}}-V_{\| \mathrm{e}}\right) \frac{1}{n}(\boldsymbol{b} \cdot \nabla) n\right] \\
+\frac{2 B}{c m_{\mathrm{i}}}\left[C\left(T_{\mathrm{e}}\right)+\frac{T_{\mathrm{e}}}{n} C(n)\right]+\frac{B}{3 c m_{\mathrm{i}} n} C\left(G_{\mathrm{i}}\right)
\end{aligned}
$$

A convenient expression for $G_{\mathrm{i}}$ still needs to be derived. From its definition, in fact, one has

$$
G_{\mathrm{i}}=-3 \eta_{0 \mathrm{i}}\left[\frac{2}{3}(\boldsymbol{b} \cdot \nabla) V_{\| \mathrm{i}}+\frac{c}{3 B} C(\phi)\right]
$$

and an analogous expression is valid for $G_{\mathrm{e}}$, that is

$$
\begin{aligned}
G_{\mathrm{e}} & =-3 \eta_{0 \mathrm{e}}\left\{\frac{2}{3}(\boldsymbol{b} \cdot \nabla) V_{\| \mathrm{e}}\right. \\
& \left.+\frac{c}{e B}\left[\frac{5}{3} C\left(T_{\mathrm{e}}\right)+\frac{5}{3} \frac{T_{\mathrm{e}}}{n} C(n)+\frac{1}{3} e C(\phi)\right]\right\}
\end{aligned}
$$

\subsection{Magnetic field}

Electromagnetic perturbations in the system introduce both compressional and shear Alfven waves. Since the compressional Alfven wave is several orders of magnitude faster than the typical turbulent fluctuations, it is important to remove it from the system. This is done by assuming that the perturbed potential vector is parallel to the background magnetic field, $\delta \boldsymbol{A}=-\psi \boldsymbol{b}_{0}$, being $\boldsymbol{b}_{0}$ the unit vector in the direction of the unperturbed magnetic field. Within the hypotheses considered for the derivation of the GBS model, in particular $\beta_{\mathrm{e}} \ll 1$, and observing that the equilibrium magnetic field varies on spatial scales longer than the fluctuation scale lengths, the perturbed magnetic field is perpendicular to $\boldsymbol{b}_{0}$ and given by

$$
\delta \boldsymbol{B}=-\nabla \times\left(\psi \boldsymbol{b}_{0}\right) \simeq \boldsymbol{b}_{0} \times \nabla_{\perp} \psi=\delta \boldsymbol{B}_{\perp} .
$$

Moreover, the Ampere's law stating the evolution of $\psi$ writes

$$
\nabla_{\perp}^{2} \psi=\frac{4 \pi}{c} j_{\|},
$$

while the electric field is given by

$$
\boldsymbol{E}=-\nabla \phi+\frac{1}{c} \frac{\partial \psi}{\partial t} \boldsymbol{b}_{0}
$$

\subsection{Motion along the magnetic field}

The parallel component of the electron momentum equation is

$$
\begin{aligned}
m_{\mathrm{e}} n & \frac{\partial V_{\| \mathrm{e}}}{\partial t}+\frac{e n}{c} \frac{\partial \psi}{\partial t}=-m_{\mathrm{e}} n \frac{c}{B}\left[\phi, V_{\| \mathrm{e}}\right]-m_{\mathrm{e}} n V_{\| \mathrm{e}}(\boldsymbol{b} \cdot \nabla) V_{\| \mathrm{e}} \\
& -\frac{2}{3}(\boldsymbol{b} \cdot \nabla) G_{\mathrm{e}}-\frac{e^{2} n^{2}}{\sigma_{\|}}\left(V_{\| \mathrm{e}}-V_{\| \mathrm{i}}\right)+e n(\boldsymbol{b} \cdot \nabla) \phi \\
& -T_{\mathrm{e}}(\boldsymbol{b} \cdot \nabla) n-1.71 n(\boldsymbol{b} \cdot \nabla) T_{\mathrm{e}}
\end{aligned}
$$

where we have used $\boldsymbol{b} \cdot \boldsymbol{\nabla} \cdot \pi_{\mathrm{e}}=2(\boldsymbol{b} \cdot \nabla) G_{\mathrm{e}} / 3$, since the FLR part of the stress tensor is perpendicular to the magnetic field. The ion momentum equation is obtained by summing the ion and electron equations and neglecting the electron inertia. One gets

$$
\begin{gathered}
m_{\mathrm{i}} n \frac{\partial V_{\| \mathrm{i}}}{\partial t}=-m_{\mathrm{i}} n \frac{c}{B}\left[\phi, V_{\| \mathrm{i}}\right]-m_{\mathrm{i}} n V_{\| \mathrm{i}}(\boldsymbol{b} \cdot \nabla) V_{\| \mathrm{i}} \\
-\frac{2}{3}(\boldsymbol{b} \cdot \nabla) G_{\mathrm{i}}-n(\boldsymbol{b} \cdot \nabla) T_{\mathrm{e}}-T_{\mathrm{e}}(\boldsymbol{b} \cdot \nabla) n .
\end{gathered}
$$

\subsection{Temperature equations}

Neglecting heat generation due to friction, collisional heat exchange, and ohmic heating due to parallel conductivity, the Braginskii equation for the electron temperature in the driftreduced limit is

$$
\begin{aligned}
\frac{\partial T_{\mathrm{e}}}{\partial t}= & -\frac{c}{B}\left[\phi, T_{\mathrm{e}}\right]-V_{\| \mathrm{e}}(\boldsymbol{b} \cdot \nabla) T_{\mathrm{e}} \\
& +\frac{4}{3} \frac{c}{e B}\left[\frac{7}{2} T_{\mathrm{e}} C\left(T_{\mathrm{e}}\right)+\frac{T_{\mathrm{e}}^{2}}{n} C(n)-e T_{\mathrm{e}} C(\phi)\right] \\
& +\frac{2}{3 e}\left\{T_{\mathrm{e}}\left[0.71(\boldsymbol{b} \cdot \nabla) V_{\| \mathrm{i}}-1.71(\boldsymbol{b} \cdot \nabla) V_{\| \mathrm{e}}\right]\right. \\
& \left.+0.71 T_{\mathrm{e}}\left(V_{\| \mathrm{i}}-V_{\| \mathrm{e}}\right) \frac{1}{n}(\boldsymbol{b} \cdot \nabla) n\right\}+S_{T},
\end{aligned}
$$

where $S_{T}$ is the plasma heat source.

\subsection{The GBS system of equations}

The model implemented in the GBS code is constituted by the continuity equation, (5), the vorticity equation, (12), Ampere's law, (16), the equation for the ion and electron parallel motion, (19) and (18), and the equation for the electron temperature, (20).

These equations are normalized as follows (tilde denotes a normalized quantity): $n=\tilde{n} n_{0}, t=\tilde{t} R / c_{\mathrm{s} 0}, \phi=$ $\tilde{\phi} T_{\mathrm{e} 0} / e, T_{\mathrm{e}}=\tilde{T}_{\mathrm{e}} T_{\mathrm{e} 0}, V_{\| \mathrm{e}}=\tilde{V}_{\| \mathrm{e}} c_{\mathrm{s} 0}, \quad V_{\| \mathrm{i}}=\tilde{V}_{\| \mathrm{i}} c_{\mathrm{s} 0}, B=$ $\tilde{B} B_{0}, \psi=\tilde{\psi} c_{\mathrm{s} 0} m_{\mathrm{i}} \beta_{\mathrm{e}} /(2 e c)$, where $n_{0}, T_{\mathrm{e} 0}, B_{0}$ are the reference density, temperature, and magnetic field, while the normalization quantities $c_{\mathrm{s} 0}$ and $\rho_{\mathrm{s} 0}$ are defined as $c_{\mathrm{s} 0}=$ $\sqrt{T_{\mathrm{e} 0} / m_{\mathrm{i}}}, \rho_{\mathrm{s} 0}=c_{\mathrm{s} 0} / \Omega_{c \mathrm{i}}$, being $\Omega_{c \mathrm{i}}=e B_{0} /\left(m_{\mathrm{i}} c\right)$. In the directions perpendicular to the magnetic field, distances are normalized to $\rho_{\mathrm{s} 0}$, while in the direction along the magnetic field the macroscopic length $R$ is used (for SMT and tokamak simulations it corresponds to the major radius). In the following, in order to simplify the notation, we drop the tildes: therefore, hereafter all the quantities are normalized. 
The normalized system of equations evolved by GBS can be written as

$$
\begin{aligned}
& \frac{\partial n}{\partial t}=-\frac{R}{\rho_{\mathrm{s} 0}} \frac{1}{B}[\phi, n]+\frac{2 n}{B}\left[C\left(T_{\mathrm{e}}\right)+\frac{T_{\mathrm{e}}}{n} C(n)-C(\phi)\right] \\
& -n(\boldsymbol{b} \cdot \nabla) V_{\| \mathrm{e}}-V_{\| \mathrm{e}}(\boldsymbol{b} \cdot \nabla) n+\mathcal{D}_{n}(n)+S \\
& \frac{\partial \omega}{\partial t}=-\frac{R}{\rho_{\mathrm{s} 0}} \frac{1}{B}[\phi, \omega]-V_{\| \mathrm{i}}(\boldsymbol{b} \cdot \nabla) \omega \\
& +B^{2}\left[(\boldsymbol{b} \cdot \nabla)\left(V_{\| \mathrm{i}}-V_{\| \mathrm{e}}\right)+\frac{\left(V_{\| \mathrm{i}}-V_{\| \mathrm{e}}\right)}{n}(\boldsymbol{b} \cdot \nabla) n\right] \\
& +2 B\left[C\left(T_{\mathrm{e}}\right)+\frac{T_{\mathrm{e}}}{n} C(n)\right]+\frac{B}{3 n} C\left(G_{i}\right)+\mathcal{D}_{\omega}(\omega) \\
& \frac{\partial V_{\| \mathrm{e}}}{\partial t}+\frac{m_{\mathrm{i}}}{m_{\mathrm{e}}} \frac{\beta_{\mathrm{e}}}{2} \frac{\partial \psi}{\partial t}=-\frac{R}{\rho_{\mathrm{s} 0}} \frac{1}{B}\left[\phi, V_{\| \mathrm{e}}\right]-V_{\| \mathrm{e}}(\boldsymbol{b} \cdot \nabla) V_{\| \mathrm{e}} \\
& -\frac{m_{\mathrm{i}}}{m_{\mathrm{e}}} \frac{2}{3}(\boldsymbol{b} \cdot \nabla) G_{\mathrm{e}}-\frac{m_{\mathrm{i}}}{m_{\mathrm{e}}} v\left(V_{\| \mathrm{e}}-V_{\| \mathrm{i}}\right)+\frac{m_{\mathrm{i}}}{m_{\mathrm{e}}}(\boldsymbol{b} \cdot \nabla) \phi \\
& -\frac{m_{\mathrm{i}} T_{\mathrm{e}}}{n m_{\mathrm{e}}}(\boldsymbol{b} \cdot \nabla) n-1.71 \frac{m_{\mathrm{i}}}{m_{\mathrm{e}}}(\boldsymbol{b} \cdot \nabla) T_{\mathrm{e}}+\mathcal{D}_{V_{\| \mathrm{e}}}\left(V_{\| \mathrm{e}}\right) \\
& \frac{\partial V_{\| \mathrm{i}}}{\partial t}=-\frac{R}{\rho_{\mathrm{s} 0}} \frac{1}{B}\left[\phi, V_{\| \mathrm{i}}\right]-V_{\| \mathrm{i}}(\boldsymbol{b} \cdot \nabla) V_{\| \mathrm{i}}-\frac{2}{3}(\boldsymbol{b} \cdot \nabla) G_{i} \\
& -\left[(\boldsymbol{b} \cdot \nabla) T_{\mathrm{e}}+\frac{T_{\mathrm{e}}}{n}(\boldsymbol{b} \cdot \nabla) n\right]+\mathcal{D}_{V_{\| \mathrm{i}}}\left(V_{\| \mathrm{i}}\right) \\
& \frac{\partial T_{\mathrm{e}}}{\partial t}=-\frac{R}{\rho_{\mathrm{s} 0}} \frac{1}{B}\left[\phi, T_{\mathrm{e}}\right]-V_{\| \mathrm{e}}(\boldsymbol{b} \cdot \nabla) T_{\mathrm{e}} \\
& +\frac{4}{3} \frac{T_{\mathrm{e}}}{B}\left[\frac{7}{2} C\left(T_{\mathrm{e}}\right)+\frac{T_{\mathrm{e}}}{n} C(n)-C(\phi)\right] \\
& +\frac{2 T_{\mathrm{e}}}{3}\left[0.71(\boldsymbol{b} \cdot \nabla) V_{\| \mathrm{i}}-1.71(\boldsymbol{b} \cdot \nabla) V_{\| \mathrm{e}}\right. \\
& \left.+0.71 \frac{\left(V_{\| \mathrm{i}}-V_{\| \mathrm{e}}\right)}{n}(\boldsymbol{b} \cdot \nabla) n\right] \\
& +\mathcal{D}_{T_{\mathrm{e}}}\left(T_{\mathrm{e}}\right)+\mathcal{D}_{T_{\mathrm{e}}}^{\|}\left(T_{\mathrm{e}}\right)+S_{T}
\end{aligned}
$$

which are coupled to Ampere's law, $\nabla_{\perp}^{2} \psi=n\left(V_{\| \mathrm{i}}-V_{\| \mathrm{e}}\right)$, and to the Poisson equation $\nabla_{\perp}^{2} \phi=\omega$. The normalised resistivity $v$ has been introduced, $v=\left(\mathrm{e}^{2} n R\right) /\left(m_{\mathrm{i}} \sigma_{\|} C_{s 0}\right)$. We also note that diffusion operators $\mathcal{D}$ and $\mathcal{D}^{\|}$have been introduced for numerical purposes: GBS allows the choice between standard diffusion and/or fourth-order hyperdiffusion operators.

We notice that an energy conservation law can be obtained starting from the GBS equations, following a development similar to the one carried out in [10]. This will be the subject of a future publication. The main differences with respect to the model in [10] come from the fact that GBS considers a simulation domain that is not periodic, therefore fluxes at the simulation boundaries have to be taken into account, as well as those from the presence of plasma and heat sources.

\subsection{Some specific models: linear devices, SMT and tokamak SOL}

The curvature operator, $C$, the perpendicular Laplacian operator, $\nabla_{\perp}^{2}$, the parallel gradient, $\boldsymbol{b} \cdot \nabla$, and the Poisson bracket, $[A, B]$, that appear in equations (21)-(25), have to be specified for each geometry; this is made easy by the
GBS modular coding. In particular, herein we focus on GBS simulations of turbulence in linear devices, in the SMT geometry, and tokamak SOL. We now provide the expressions for the operators in these three geometries.

According to typical experimental data, plasma turbulence in linear machines, such as LAPD, can be described by considering the electrostatic limit. A coordinate system is chosen such that $x$ and $y$ are coordinates in the perpendicular plane, and $z$ is the parallel direction. The operators assume a particularly simple form: $C=0,[A, B]=\partial_{x} B \partial_{y} A-$ $\partial_{x} A \partial_{y} B, \nabla_{\perp}^{2}=\partial_{x}^{2}+\partial_{y}^{2}$, and $\boldsymbol{b} \cdot \boldsymbol{\nabla}=\partial_{z}$.

For SMT and tokamak SOL simulations, we consider a coordinate system where we denote the perpendicular coordinates with $x$ and $y$, being $x$ the radial coordinate, and $y$ the coordinate perpendicular to both $x$ and the magnetic field. We use $z$ for the periodic toroidal direction, such that $0<z<2 \pi$. In the SMT, where electrostatic turbulence is considered, the expression for the operators is $C=\partial_{y}$, $[A, B]=\partial_{x} B \partial_{y} A-\partial_{x} A \partial_{y} B, \nabla_{\perp}^{2}=\partial_{x}^{2}+\partial_{y}^{2}$, and

$$
\boldsymbol{b} \cdot \boldsymbol{\nabla}=\frac{\partial}{\partial z}+\frac{L_{v}}{2 \pi N R} \frac{\partial}{\partial y},
$$

where $N$ is the number of field line turns in the SMT, $R$ its major radius, and $L_{v}$ is the height of the device.

On the other hand, for tokamak SOL turbulence, herein we consider electromagnetic turbulence in configurations with circular magnetic flux surfaces, a toroidal limiter on the highfield side of the simulation domain, and no magnetic shear (a discussion on the impact of magnetic shear on SOL linear modes is in [52]). In this case we set $C=-\sin \theta \partial_{x}-\cos \theta \partial_{y}$, $[A, B]=\partial_{x} B \partial_{y} A-\partial_{x} A \partial_{y} B, \nabla_{\perp}^{2}=\partial_{x}^{2}+\partial_{y}^{2}$, and $\boldsymbol{b} \cdot \nabla A=$ $\boldsymbol{b}_{0} \cdot \nabla A+\beta[\psi, A] /(2 B)$, being

$$
\boldsymbol{b}_{0} \cdot \nabla=\frac{\partial}{\partial z}+\frac{a}{R q} \frac{\partial}{\partial y},
$$

$q$ the safety factor, and $\theta=y / a$ the poloidal angle defined so that $\theta=0$ and $\theta=2 \pi$ at the equatorial high-field side midplane. We note that the term $[\psi, A]$ represents the component of the parallel gradient of $A$ along the turbulent perturbations of the magnetic field.

In the simulations presented in this paper, we consider standard perpendicular and parallel diffusion operators, e.g. $\mathcal{D}=\partial_{x}^{2}+\partial_{y}^{2}$. The source term is set to mimic the plasma production process specific to each device. For example, for the LAPD linear machine [40], where plasma is produced by a plasma cathode, a top-hat density source has been chosen, which is uniform along the axial direction. For the TORPEX SMT, two Gaussian sources have been implemented in order to simulate the plasma sources at the electron-cyclotron and upper-hybrid resonance layers [53]. Finally, in the SOL configuration, a radially localized Gaussian source models the particle and heat outflow from the tokamak core.

\subsection{Boundary and initial conditions}

GBS allows the choice between Neumann and Dirichlet boundary conditions for all the fields. For $V_{\| \mathrm{i}}$ and $V_{\| \mathrm{e}}$ we 
have also implemented Bohm's boundary conditions, which state that $V_{\| \mathrm{i}}=\sqrt{T_{\mathrm{e}}}$, and $V_{\| \mathrm{e}}=\sqrt{T_{\mathrm{e}}} \exp \left(\Lambda-\phi / T_{\mathrm{e}}\right)$ at the plasma sheath edge, where $\Lambda=\log \sqrt{m_{\mathrm{i}} /\left(2 \pi m_{\mathrm{e}}\right)} \simeq 3$ [3].

When a new simulation is started, each quantity $A$ is initialized according to $A\left(t_{0}\right)=A_{0}+\tilde{A}(x, y, z)$, where $A_{0}$ is a constant value, and $\tilde{A}(x, y, z)$ is a random field whose amplitude can be chosen. Sources inject plasma particles and heat, steepening the plasma profile and triggering plasmagradient driven instabilities. The wavelength of the instability that dominates the simulations matches the fastest growing instability evaluated with a linear stability code. After a transient phase, a quasistationary state is reached in which the plasma, generated by the source and transported by turbulence, is eventually removed from the system by losses at the vessel walls. The analysis is typically focused on this quasi-stationary phase.

\section{The GBS code}

\subsection{Numerical algorithm}

In GBS, the domain is discretized using $N_{x}, N_{y}, N_{z}$ intervals in the $x, y, z$ direction, respectively. We define the grid point $x_{i}=(i-1 / 2) \Delta x$, for $i=0, . ., N_{x}+1$, the width of intervals being $\Delta x=L_{x} / N_{x}$, and the points corresponding to $i=0$ and $i=N_{x}+1$ therefore representing the ghost cells. Analogous expressions are valid in the $y$ direction, i.e. $y_{j}=(j-1 / 2) \Delta y$. Regarding the $z$ direction, we remark that $L_{z}=2 \pi$ for the SMT and the tokamak geometry, and that the grid is defined for the $n, T_{\mathrm{e}}, \phi$, and $\omega$ variables as $z_{k}=k \Delta z$ while, for numerical reasons, it is shifted by half a cell for $V_{\| \mathrm{i}}, V_{\| \mathrm{e}}$, and $\psi$, i.e. $z_{k}=(k-1 / 2) \Delta z$. Each physical quantity $A(x, y, z)$ is written as $A_{i j k} \equiv A\left(x_{i}, y_{j}, z_{k}\right)$.

The derivatives in the $x, y$ directions are performed with a standard centered finite-difference scheme, e.g.

$$
\frac{\partial}{\partial x} A(x, y, z) \simeq \frac{A_{i+1, j, k}-A_{i-1, j, k}}{2 \Delta x}
$$

except for the Poisson bracket terms, which are discretized according to the Arakawa scheme [54]. Regarding the parallel gradient, in the case of a linear machine it reduces to the derivative along the $z$ direction and can be treated with standard centered finite-difference scheme. On the other hand, it has to be approached with particular care in the SMT and SOL cases. In these configurations the discretization of the operator in equations (26) and (27) has to take advantage of the fact that turbulence is mostly aligned with the field lines in order to reduce the computational cost of the simulations. In GBS we align the $(y, z)$ grid with the field lines, such that the resolution along $z$ can remain low. For this purpose, for the SOL we choose the number of grid points, $N_{y}$ and $N_{z}$, in such a way that $\Delta j=N_{y} /\left(q N_{z}\right)$ is an integer, where $\Delta j$ represents for a field line the shift of grid points along the $y$ direction in correspondence to a $z$ shift of one grid point (analogous expressions are valid for the SMT). We then approximate the parallel derivative as

$\left.\boldsymbol{b}_{0} \cdot \nabla A\right|_{x_{i}, y_{j}, z_{k}} \simeq \frac{1}{2 \Delta z}\left(A_{i, j+\Delta j, k+1}-A_{i, j-\Delta j, k-1}.\right)$.
Table 1. Numerical convergence test with the number of spatial grid points for a SOL simulation with $\beta_{\mathrm{e}}=8 \times 10^{-4}, v=0.1, q=4$, $m_{\mathrm{i}} / m_{\mathrm{e}}=200, L_{y}=800, R=1000$.

\begin{tabular}{llll}
\hline & $N_{x}=128$ & $N_{x}=128$ & $N_{x}=128$ \\
& $N_{y}=512$ & $N_{y}=512$ & $N_{y}=1024$ \\
& $N_{z}=32$ & $N_{z}=64$ & $N_{z}=64$ \\
\hline$R / L_{n}$ & 9.28 & 10.13 & 10.67 \\
$R / L_{T}$ & 5.48 & 5.44 & 5.41 \\
\hline
\end{tabular}

In doing this, care must be taken in considering that the parallel velocities are evaluated corresponding to a different $z$ grid with respect to the density, temperature and potential. This scheme allows us to consider only safety factors that take integer values. We remark that, unlike in the closed flux surface region, this does not constitute an important limitation in exploring the SOL physics since the magnetic field lines are open and terminate on the vessel. Therefore $q$ has only a geometrical meaning in the SOL, related to the ratio between the toroidal and poloidal components of the magnetic field [3].

The Laplacian operator in the Poisson equation is discretized using a standard second-order finite difference. Depending on the geometry, the obtained matrix can be solved by direct matrix inversion or can be reduced to the solution of a set of tridiagonal systems, by applying the fast Fourier transform (FFT) algorithm in one direction.

In order to time advance the fluid equations we use a standard fourth-order Runge-Kutta scheme. An adaptative time stepping scheme has also been implemented, which is a third-order Runge-Kutta scheme with an embedded secondorder method used to control the local integration error [55]. Finally, we note that the equations implemented in GBS are rewritten in terms of $\theta_{n}=\log n$ and $t_{\mathrm{e}}=\log T_{\mathrm{e}}$, to ensure the positivity of $n$ and $T_{\mathrm{e}}$.

Numerical tests have been performed to check the convergence properties of the GBS numerical method with respect to the time step and the spatial grid. First, by testing separately each discretized operator against analytical solutions, we confirmed that the error due to the time discretization scales as $\Delta t^{4}$, that the error affecting the discretization of the perpendicular directions scales as $\Delta x^{2}$ and $\Delta y^{2}$, while the numerical error affecting the parallel derivative scales as $\Delta z^{2}$. Second, we have carried out convergence tests of the GBS turbulence results by considering, because of the chaotic features of turbulence, time-averaged properties. For a SOL simulation, the results of one of these tests are shown in table 1, which displays the normalized time- and $y$-averaged density and temperature scale lengths for three different computational grids. Similar convergence tests have been performed with the temporal discretization.

\subsection{Parallelization}

GBS is parallelized with a domain decomposition technique using MPI. The physical domain in the $x$ direction is equally divided into $N_{P_{x}}$ parts and the physical domain in the $z$ direction is equally divided into $N_{P_{z}}$ parts; the total number of processes is therefore $N_{P}=N_{P_{x}} N_{P_{z}}$. In each process, in the $x$ and $z$ 


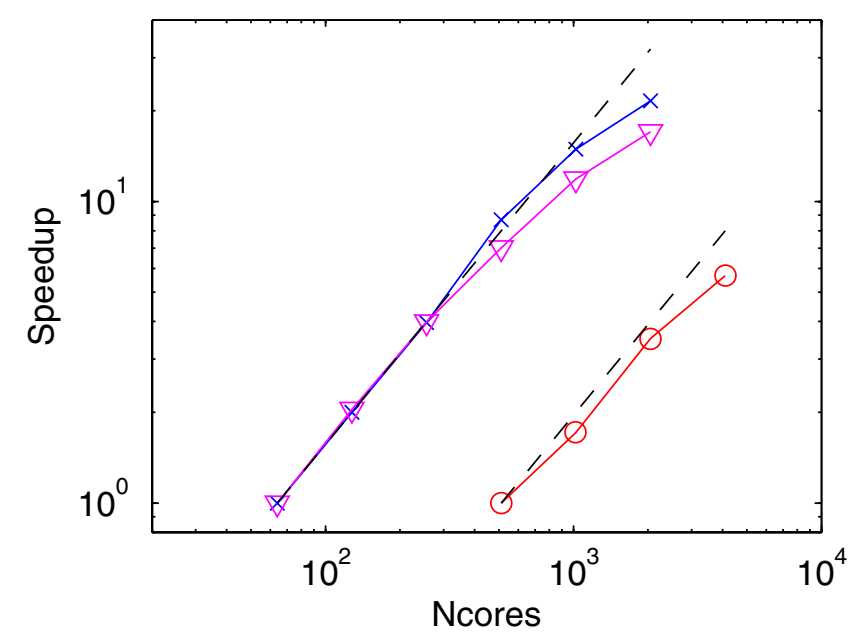

Figure 1. GBS speed up on a Cray XT6 using the FFT transform (blue crosses) and the MUMPS library (magenta triangles), and on a BlueGene/P with FFT transform (red circles). Ideal scalings are indicated with the dashed lines.

directions, one ghost cell must be added to both sides of the domain. As a consequence, an array representing a physical quantity will have a size of $\left(N_{x} / N_{P_{x}}+2\right)\left(N_{y}+2\right)\left(N_{z} / P_{z}+2\right)$ elements in each process.

Message passing among the different processes is required for updating the ghost cells and for the solution of the Poisson equation. For each array $A_{i j k}$, each process must communicate $\left(N_{x} / N_{P_{x}}+2\right)\left(N_{y}+2\right)$ elements to the two neighboring processes in the $z$ communicator and $\left(N_{z} / N_{P_{z}}+\right.$ 2) $\left(N_{y}+2\right)$ elements to the two neighboring processes in the $x$ communicator. The boundary conditions for $V_{\| \mathrm{e}}$ and $V_{\| \mathrm{i}}$ require some additional communications if Bohm-like boundary conditions are chosen.

At each time step, the Poisson equation is solved to obtain $\phi$ (and a second Poisson equation has to be solved to obtain $\psi$, in the case of electromagnetic simulations). In the case that the direct matrix inversion is performed, this is accomplished by using parallel libraries like MUMPS [56], Pardiso [57, 58], or LAPACK [59]. Within the FFT algorithm, a parallel transpose operation is performed in order to perform the FFT and the tridiagonal matrix inversion on the same processor.

To provide an idea of the numerical cost of a GBS simulation, we notice that the largest SOL GBS simulations are run on a grid of $2048 \times 128 \times 128$ points in the poloidal, toroidal, and radial direction, respectively. Scaling tests performed on a Cray XT6 machine have shown that one time step can be carried out in 0.5 seconds on 1024 processors. Since the typical time step is of the order of $10^{-4}$ and in order to get steady state simulations one has to cover a time span of the order of 70 , $10^{5} \mathrm{CPU}$ hours are necessary to perform one simulation. The GBS strong scaling performances are shown in figure 1.

\section{Simulation results}

\subsection{Linear devices}

GBS simulations have been performed using LAPD-like parameters [40]. LAPD is a device that creates a linear plasma

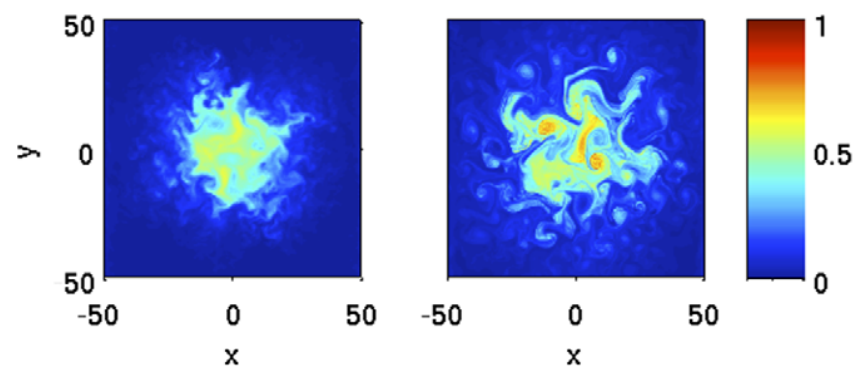

Figure 2. Snapshot of plasma density in a linear device. The plane perpendicular to the magnetic field is displayed. The three-dimensional simulation (left) is compared with the two-dimensional one (right). The similar level of transport shown by the two simulations reveals that $k_{\|}=0$ modes are those dominating the plasma dynamics.

approximately $18 \mathrm{~m}$ long and $0.3 \mathrm{~m}$ in radius with straight magnetic field lines that terminate on the end walls. Among its many uses, this experiment has been applied to the study of turbulence and transport [60-64].

Global three-dimensional two-fluid simulations of turbulence in a linear device with LAPD-like plasma parameters and Bohm sheath boundary conditions in the parallel direction are presented in [36]. The GBS simulation results are somewhat unexpected: it was found that drift waves, although present, are not the main source of heat and particle cross-field transport in the device. Nor are sheath-driven instabilities [65], which arise in the system from the sheath (Bohm) boundary conditions in the parallel direction [3]. Rather, the main agent of transport is the Kelvin-Helmholtz (KH) instability. This is a fully global mode, driven by shear in the equilibrium electrostatic potential arising from the sheath boundary conditions [3]: $e \phi \simeq \Lambda T_{\mathrm{e}}$. The nonlinear evolution of the $\mathrm{KH}$ mode produces large-scale eddies that are the main sources of cross-field profile relaxation.

This finding has been tested by comparing the results of three-dimensional and two-dimensional simulations. Since the two-dimensional simulations assume $k_{\|}=0$, preventing the growth of the drift waves, the similarity of their results with respect to the ones from three-dimensional simulations confirms that drift waves do not play a major role in the plasma dynamics. Moreover, tests where the drive of the $\mathrm{KH}$ instability is removed from the system show a strong suppression of the turbulence, confirming the nature of the plasma dynamics.

Typical turbulence snapshots are shown in figure 2 . The largest perpendicular structures correspond to the most unstable $\mathrm{KH}$ modes in the system. These are global modes with a radial extent comparable to $L_{0}$, the radial gradient scale length of $\phi$, poloidal wavenumbers $k_{\theta} L_{0} \sim 1$, and $k_{\|} \simeq 0$. The shorter scale activity in the plots is produced by drift waves and, to a lesser extent, sheath modes.

\section{2. $S M T$}

The SMT configuration is characterized by the toroidal and vertical components of the magnetic field, $B_{\varphi}$ and $B_{v}$, which create helicoidal field lines that terminate on the upper and lower walls of the torus vessel $[41,42,66-72]$. The goal 


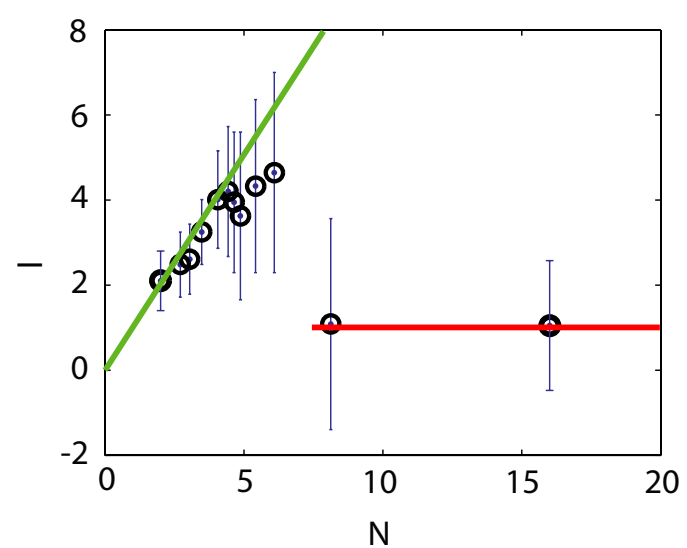

Figure 3. The vertical mode number, $l$, is shown as function of $N$, the number of field line turns in an SMT. Circles denote the TORPEX experimental results and their relative error bars, while solid lines interpolate the GBS results, which point out the presence of the ideal-interchange mode for $N \lesssim 7$ (green line) and the resistive interchange mode for $N \gtrsim 7$ (red line).

of SMT experiments is to allow detailed studies of plasma turbulence in a configuration that, like the SOL of magnetic fusion devices, features open field lines, magnetic curvature, and plasma gradients.

Experiments show that SMT plasma turbulence presents a number of turbulent regimes. The presence of idealinterchange instabilities with $k_{\|}=0$ has been noted in a number of studies (see, e.g. [71,72]), as well as $k_{\|} \neq 0$ instabilities, typically interpreted as drift waves (see, e.g. [7375]). The transition from $k_{\|}=0$ ideal-interchange mode dominated turbulence to a finite $k_{\|} \neq 0$ state is observed in the TORPEX device as the pitch angle of the field lines with respect to the upper and lower walls is decreased [76, 77]. The pitch can be expressed in terms of $N$, the total number of field line turns in the SMT. Over one toroidal circuit around the machine, the field lines are displaced vertically by $\Delta=2 \pi R B_{v} / B_{\varphi}$, where $R$ is the major radius. Denoting the height of the vacuum vessel by $L_{v}$, the number of turns from bottom to top is thus $N=L_{v} / \Delta=L_{v} B_{\varphi} /\left(2 \pi R B_{v}\right)$. The pitch decreases as $N$ is increased, and the onset of finite $k_{\|} \neq 0$ fluctuations occurs when $N$ becomes sufficiently large. Based on TORPEX measurements, in figure 3, the vertical mode number $l$ (corresponding to a vertical wavenumber $\left.k_{v}=2 \pi l / L_{v}\right)$ is plotted as a function of $N$. The $k_{\|}=0$ regime, in which $l=N$, is observed for small $N \lesssim 7$. The dominant toroidal mode number in this case is $n=1$, the expected value given $k_{\|}=0$ and $l=N$. For $N \gtrsim 7$ the turbulence transitions to a state dominated by $l=1$ fluctuations (one wavelength in the vertical direction from bottom to top), which corresponds to a small but finite $k_{\|}=1 /(R N)$. The dominant toroidal mode number in this regime is $n=0$; that is, the turbulence becomes toroidally symmetric.

Using GBS simulations, it has been possible to predict the character of the SMT turbulence as a function of $N$ and other parameters [35]. Focusing on cases in which shear flow plays a minor role, GBS simulations show that three turbulent regimes are present in the SMT: (i) an idealinterchange regime characterized by $k_{\|}=0, l=N$ and
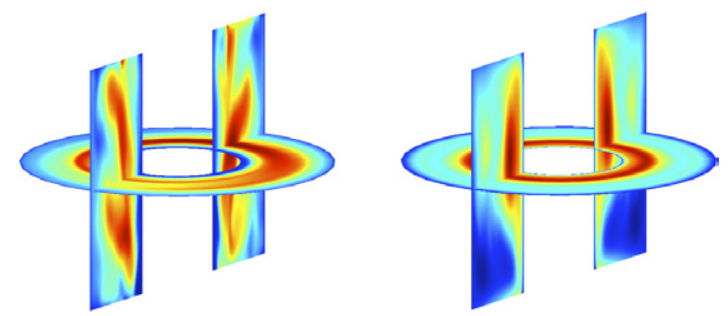

Figure 4. Snapshots of plasma turbulence in the SMT configuration. The electrostatic potential $\phi$ is displayed on two poloidal cross sections and on the toroidal midplane. On the left panel, we show an $N=2$ case, with $k_{\|}=0$ ideal-interchange dominated turbulence. On the right panel, the $N=16$ configuration displays the typical dynamics dominated by the resistive interchange modes with $k_{\|} \neq 0$ and $l=1$.

$n=1$; (ii) a resistive interchange regime with $k_{\|} \neq 0$, $n=0$, and $l=1$; and (iii) a drift-wave regime that is obtained for sufficiently steep gradients $L_{p}<L_{p \text {, crit }}$ and is characterized by short vertical wavelengths, $k_{\perp} \rho_{s} \sim 0.5$. At the relatively high electron-neutral collisionality typical of SMT experiments, GBS simulations suggest that the transport driven by interchange modes prevents the gradient scale lengths from steepening into the drift-wave dominated regime. The behavior of $l$ in figure 3 therefore reflects a transition from ideal-interchange mode turbulence to a state dominated by resistive interchange instabilities (see figure 4).

The resistive interchange modes in the SMT are similar to resistive ballooning modes in the edge region of tokamaks: they have maximum growth rates comparable to the idealinterchange mode $\gamma^{2} \sim c_{\mathrm{s}}^{2} /\left(R L_{p}\right)$ and occur at given parallel and perpendicular wavenumbers when the conductivity is sufficiently small. The instability threshold follows from the vorticity equation, (22): the polarization drift term $\partial_{t} \nabla_{\perp}^{2} \phi$ must exceed the line bending term proportional to $\nabla_{\|} j_{\|}$; with Ohm's law, equation (23), $j_{\|} \sim-\sigma_{\|} \nabla_{\|} \phi$, this condition yields $\gamma k_{\perp}^{2}>4 \pi V_{A}^{2} k_{\|}^{2} \sigma_{\|} / c^{2}$. Because $k_{\|}^{2}=1 /(N R)^{2}$, line bending becomes negligible for sufficiently high $N$. Since $k_{\perp} \sim 2 \pi / L_{v}$ $(l=1)$, the numerical study of such modes requires global simulations that cover the entire SMT domain, which were performed for the first time with GBS.

GBS has also been used to simulate the impact of an externally imposed bias voltage on the interchange-driven turbulence and transport in the SMT device [38]. This study is inspired by the bias experiments performed on the Helimak SMT device [69], and is focused on the idealinterchange instability regime. In the grounded case, the turbulent structures of the density and temperature driven by the interchange mode are bursty, and have relatively wide radial extents on the low-field side. In the biased case, a bias voltage is applied to the upper and lower boundaries over a small radial extension. The structures of plasma potential are changed in response to the bias voltage, resulting in equilibrium sheared flows. These bias-induced vertical flows located in the region with steep pressure gradients reduce the radial extent of turbulent structures, and lower convective transport of particles and heat on the low-field side, consistent with Helimak observations. 


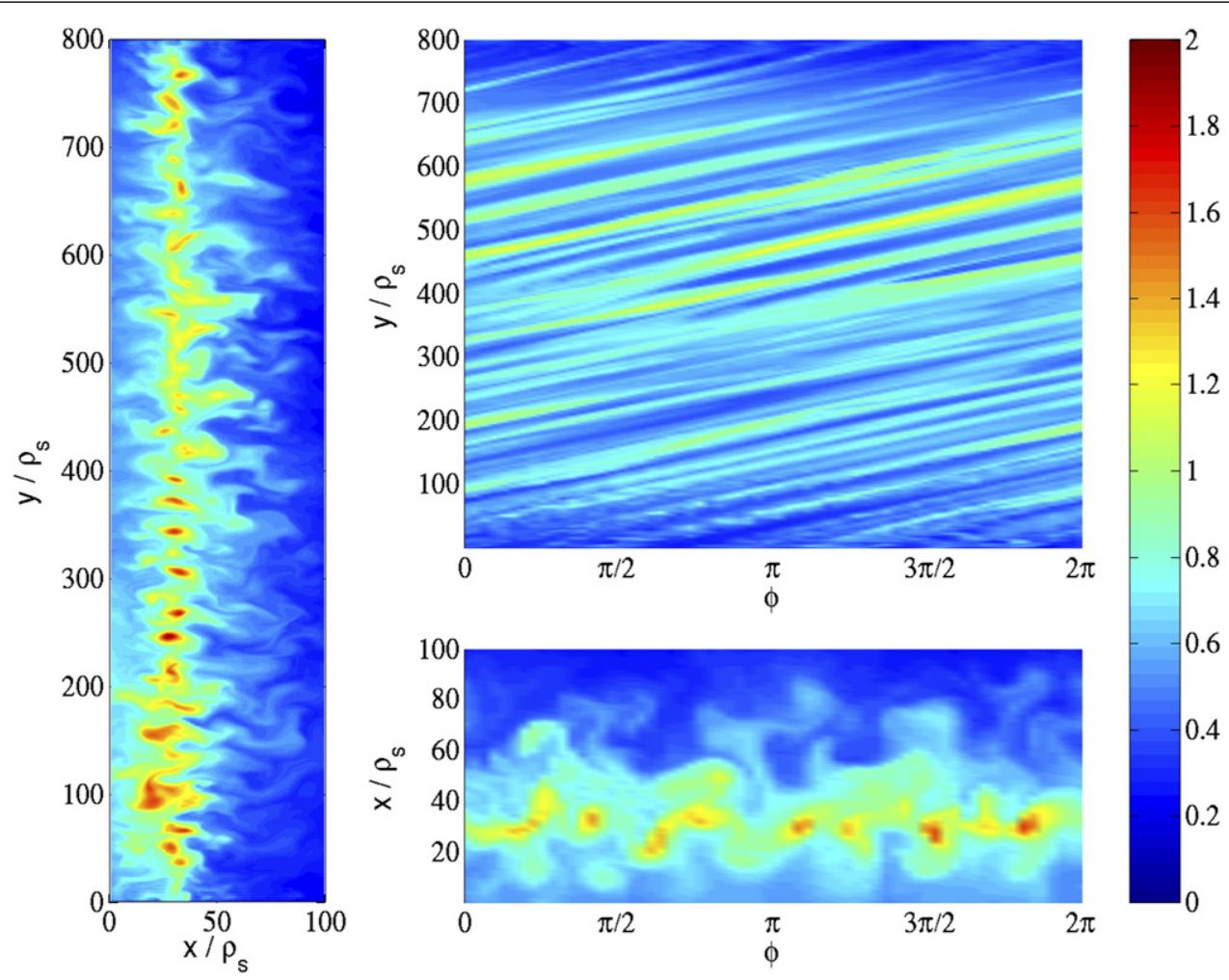

Figure 5. Snapshot of electron pressure in the SOL. A poloidal plane is represented on the left panel, while a radial cut and a toroidal cut are shown on the right upper panel and right lower panel, respectively.

\subsection{Tokamak SOL}

Herein we report the first simulations of SOL turbulent dynamics that have been carried out with GBS. We have considered a relatively simple tokamak configuration, with circular flux surfaces, neglecting finite aspect ratio terms and magnetic shear, and with a toroidal limiter located at the equatorial plane, on the high-field side of the machine.

We show a snapshot of turbulence, displaying the plasma pressure, $p_{\mathrm{e}}$, and electrostatic potential, $\phi$, in figures 5 and 6 . We consider the projections on the poloidal, toroidal and radial planes. The plasma dynamics that is displayed is the result of the interplay between plasma outflow from the tokamak core, turbulent transport, and losses at the limiter plates, which are located in correspondence of $y=0$ and $y=800$. Bohm's boundary conditions are implemented at the limiter plates imposing $\phi=\Lambda T_{\mathrm{e}}$.

The plasma outflowing from the core is modeled as density and temperature sources located at $x=30$, uniform in the $y$ direction and with a Gaussian shape in the radial direction, with half width $w=5$. Therefore, the region of interest in the simulation to model the SOL turbulence is for $x>30$. From the source region, plasma is transported in the radial direction by turbulence. In the present SOL simulation, turbulence results from the growth of the resistive ballooning mode that, as it is visible in the turbulent snapshots in figures 5 and 6 , has the longest parallel wavelength allowed by the system, $k_{\|} \sim 1 /(q R)$. The poloidal mode number, $m$, is constrained between a lower limit, due to the field-line bending as for the resistive interchange mode in the SOL, and an upper limit due to curvature-induced plasma compressibility [50]. The toroidal mode number results from $n \simeq m / q$. According to the ballooning nature of the instability, turbulence is more active for $y>400$ : in fact, it is driven in the unfavorable curvature region on the low-field side of the SOL and then it is convected vertically upward by the $\boldsymbol{E} \times \boldsymbol{B}$ drift.

Although relatively simple, the present model allows us to identify some of the most prominent elements that characterize SOL turbulence measurements. In fact, turbulence presents a coherent dynamics close to the source region while it is dominated in the far SOL by intermittent transport events. Those are the so-called plasma blobs, which have attracted great attention from the plasma physics community since they can be detrimental for the tokamak first wall [78]. Through GBS simulations it is possible to study their dynamics, from their birth to the dissipation on the limiter plates. In general, it is observed that fluctuations have an amplitude comparable to that of equilibrium quantities, and that there is no separation of fluctuation and equilibrium scales. This makes it necessary to treat turbulence globally, as carried out by GBS.

\section{Code validation}

Validation of plasma turbulence codes plays a fundamental role in assessing the maturity of the understanding of plasma dynamics and the predictive capabilities of simulations. A validation project is a four-step procedure [79,80]. First (i), the simulation model needs to be qualified, i.e. it is necessary to establish the applicability of the model hypotheses for the simulated physical phenomenon. Second (ii), verification of the code is necessary, in order to prove 

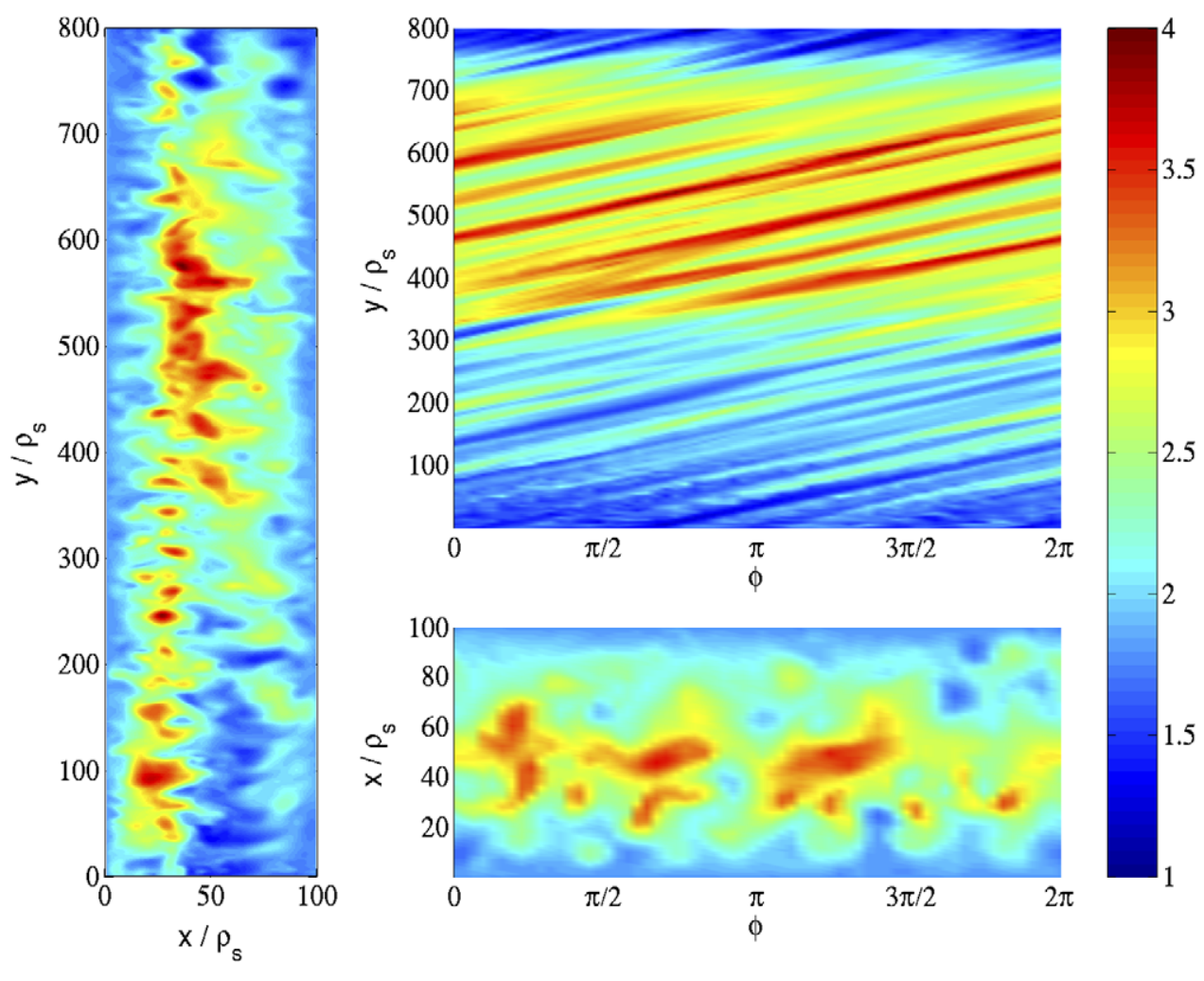

Figure 6. Same as in figure 5, for electrostatic potential.

that the code solves correctly the model equations. Third (iii), simulations and experiments have to be compared considering a number of physical quantities, common to the experimental measurements and simulation results, and analyzed using the same techniques. These physical quantities, denoted as validation observables, should be identified and organized into a hierarchy. This hierarchy is based on the number of model assumptions and combinations of measurements necessary to obtain the observable; i.e. how stringent each observable is for comparison purposes. Fourth (iv), agreement between simulations and experiments needs to be quantified by using an appropriate composite metric, $\chi$. This $\chi$ coefficient should combine the results of the comparison of all the observables, taking into account position in the hierarchy and precision. Its purpose is to quantify the overall agreement between experiment and simulation. The metric $\chi$ should be complemented by an index, $Q$, which assesses the quality of the comparison. Practically, $Q$ quantifies the number of observables that have been used for the validation and the strength of the constraints they impose. We remark that the validation procedures should remain simple. The goal is not mathematical rigor, but a useful tool that can be easily applied in order to compare models and assess their predictive capabilities and their limitations.

While model qualification and code verification (points (i) and (ii) of the validation guidelines) are now routinely considered in plasma physics and their methodology has been formulated in considerable detail, only recently has the plasma physics community approached a rigorous methodology for establishing the validation observables and the comparison metric. With the development of the GBS code, we have carried out a validation project with the goal of defining the methodology for the comparison of experimental and simulation results, and we have then applied it to the analysis of the TORPEX device [31,37].

In [31], we address point (iii) of the validation methodology. Focusing on observables related to Langmuir probe measurements on TORPEX plasmas, we consider a number of physical quantities that can be used as observables for experiment/simulation comparison. We classify the observables according to a hierarchy that sums the number of model assumptions and measurement combinations used to obtain an observable from experimental measurements and simulation results. Observables in this hierarchy go from the measurement of the ion saturation current, which is a direct experimental measurement and can be inferred directly from density and temperature simulation data, to the measurement of particle transport, which requires the combination of a number of measurements with hypotheses that also challenge the analysis of simulation data. The more assumptions needed, the less stringent the comparison with respect to this observable and, thus, its weight in the evaluation of the agreement between experiments and simulations should be decreased. We also point out that one has to use the same techniques to analyze experimental and simulation data, in order to directly compare the experimental and simulation values of each observable.

The construction of a global metric in order to compare experiments and simulations and the definition of the comparison quality, $Q$, point (iv) of the validation methodology, are the subject of [37]. A key step in the construction of the global metric is the quantification of the agreement between experiment and simulation relative 
to each observable. We denote with $e_{j}$ and $s_{j}$ the values of the $j$ th observable used in the comparison, as coming from the experimental measurement or the simulation results, respectively. Most of the observables depend on space and time, and typically the value of the observables is given on a discrete number of points, denoted as $N_{j}$. We denote with $e_{j, i}$ and $s_{j, i}$ the values of the $j$ th observable at points $i=1,2, \ldots, N_{j}$ (the present notation can therefore be used for zero-, one-, two-, etc, dimensional observables). For the $j$-th observable, we normalize the distance $d_{j}$ between experiments and simulations with respect to the uncertainty related to these quantities:

$$
d_{j}=\sqrt{\frac{1}{N_{j}} \sum_{i=1}^{N_{j}} \frac{\left(e_{j, i}-s_{j, i}\right)^{2}}{\Delta e_{j, i}^{2}+\Delta s_{j, i}^{2}}},
$$

where $\Delta e_{j, i}$ and $\Delta s_{j, i}$ are the uncertainties related to the evaluation of $e_{j, i}$ and $s_{j, i}$. Since simulations and experiments can be considered to agree if they fall within the error bars, we define the level of agreement between experiments and simulations with respect to observable $j$ as

$$
R_{j}=\frac{\tanh \left[\left(d_{j}-d_{0}\right) / \lambda\right]+1}{2}
$$

with $R_{j} \lesssim 0.5$ corresponding to agreement (within the experimental and simulation uncertainties), while $R_{j} \gtrsim$ 0.5 denoting disagreement (outside the experimental and simulation uncertainties). Our tests show that the conclusions of a validation exercise are not affected by the specific choices of parameters in the range $1 \leqslant d_{0} \leqslant 2$ and $0.1 \leqslant \lambda \leqslant 1$, and we believe that the values $d_{0}=1.5$ and $\lambda=0.5$ are reasonable choices.

The overall level of agreement between simulations and experiments can be measured by considering a composite metric, which should take into account the level of agreement of each observable, $R_{j}$, and weight it according to how constraining each observable is for comparison purposes. This means that the hierarchy level of each observable and the level of confidence characterizing the measurement or the simulation of each observable have to be taken into account. The higher the level in the primacy hierarchy and the bigger the error affecting the observable measurement, the smaller the weight of the observable should be. We thus define the metric $\chi$ as

$$
\chi=\frac{\sum_{j} R_{j} H_{j} S_{j}}{\sum_{j} H_{j} S_{j}},
$$

where $H_{j}$ and $S_{j}$ are functions defining the weight of each observable according to its hierarchy level and the precision of the measurement, respectively. Thanks to the definition of $R_{j}, \chi$ is normalized in such a way that perfect agreement is observed for $\chi=0$ (within the considered observables), while simulation and experiment disagree completely for $\chi=1$.

The definition of $H_{j}$ and $S_{j}$ is somewhat arbitrary. $H_{j}$ should be a decreasing function of hierarchy levels. The definition we adopt is $H_{j}=1 / h_{j}$, where $h_{j}$ is the combined experimental/simulation primacy hierarchy level (see [31] for more details on the $h_{j}$ definition). This definition implies that if no assumptions or combinations of measurements are used for obtaining an observable, then $H_{j}=1$. The quantity $S_{j}$ should be a decreasing function of the experimental and simulation uncertainty. We introduce the following definition:

$$
S_{j}=\exp \left(-\frac{\sum_{i} \Delta e_{j, i}+\sum_{i} \Delta s_{j, i}}{\sum_{i}\left|e_{j, i}\right|+\sum_{i}\left|s_{j, i}\right|}\right)
$$

such that $S_{j}=1$ in the case of zero uncertainty.

The validation metric should be complemented by an index, $Q$, that assesses the "quality" of the comparison. The idea is that a validation is more reliable with a larger number of independent observables, particularly if they occupy a low level in the primacy hierarchy and the measurement and simulation uncertainties are low. The quality of the comparison $Q$ can thus be defined as

$$
Q=\sum_{j} H_{j} S_{j}
$$

We have applied the validation procedure to the study of the TORPEX device. Owing to its detailed diagnostics, possibility of parameter scans, and relatively simple configuration, TORPEX is an ideal testbed to perform experiment/simulation comparisons and to investigate the corresponding methodological framework [41, 42]. As shown in figure 7, we perform the comparison between experiments and simulations through a scan in the $N$ parameter, allowing the properties of the TORPEX turbulence to pass from $k_{\|}=0$ mode dominated turbulence to $k_{\|} \neq 0$ turbulence. Using a relevant set of observables, we validate two models: threedimensional GBS simulations, able to describe the global evolution of TORPEX plasma [35], and the reduced twodimensional GBS simulations [30], able to describe only the evolution of $k_{\|}=0$ modes. We show that the validation metric is able to point out that the agreement of the two-dimensional simulations and the experiment is no longer satisfactory when $k_{\|} \neq 0$ modes are present in the experiment, which occurs at $N \gtrsim 7$. A detailed analysis of the agreement with respect to each observable used for the comparison is presented in $[31,37]$ and it points out that GBS is able to well predict the modes that dominate the transport, while it overestimates the plasma pressure scale length. Possible GBS improvements that could lead to a better agreement between GBS results and experiments are discussed in [37].

We believe that the proposed methodology can be easily applied to discriminate among models, since a smaller $\chi$ corresponds to a model or a code that provides a better global representation of the physical phenomena at play. Moreover, the proposed methodology is very useful to check how the agreement varies with the control parameters. In fact, the increase of $\chi$ that follows from a variation of a control parameter points out the presence of a regime where a particular model is not appropriate, presumably because at least one physical phenomenon is not well captured. Thus, the most direct use of the proposed validation methodology is related to determining which code has to be used, and in which parameter regime. On the other hand, while it is relatively easy to discriminate among models and the different parameter regimes, it is much more difficult to judge a model in absolute terms. 

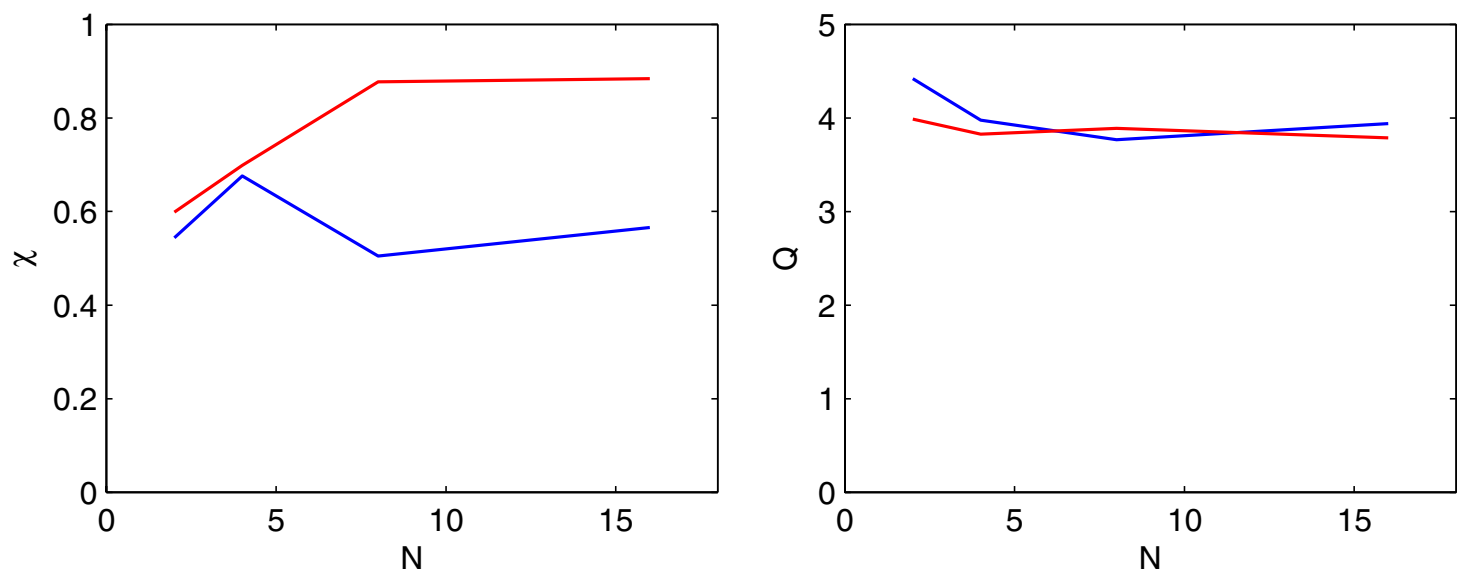

Figure 7. The results of the GBS validation against TORPEX experimental data are shown. The global metric, $\chi$, and the quality of the validation, $Q$, is shown for the three-dimensional GBS simulations (blue line) and the two-dimensional one (red line), as a function of $N$ the number of field line turns in the SMT.

\section{Conclusions and outlook}

In this paper we have described the GBS code, a tool to simulate plasma turbulent dynamics in the tokamak SOL. Based on the drift-reduced Braginskii equations, GBS is able to model SOL plasma turbulence as the interplay between the plasma outflow from the tokamak core, the turbulent transport, and the losses at the limiter plates. GBS development has been progressive: GBS has been first developed to model turbulence in basic plasma physics devices, such as linear devices and SMT, which contain some of the main elements of SOL turbulence but in a simplified setting. In this paper we have summarized the findings obtained from the simulation carried out in these configurations. The code has then been extended to the SOL geometry. We have reported the first simulations of SOL turbulence. We have considered electromagnetic turbulence with circular flux surfaces, neglecting finite aspect ratio terms and magnetic shear, and with a toroidal limiter located at the high-field side of the machine.

While GBS is still being applied to carry out simulations of basic plasma experiments, including other devices than the ones mentioned here, for instance the HelCat device [81], progress is being made in order to represent more accurately the SOL configuration. In particular, we are introducing in GBS finite aspect ratio effects, magnetic shear, and finite $T_{\mathrm{i}}$ effects. We are also currently investigating the boundary conditions at the limiter plates, in order to take into account the physics at the sheath edge more accurately in our simulations [82]. Our goal is to use the GBS code to study how SOL turbulence scales with tokamak size, understanding the role of magnetic shear and $\beta$ and, ultimately, to obtain a diagram of the SOL turbulence regimes depending on the tokamak operating parameters. In the long term, our goal is to couple SOL turbulence simulations with the simulation of plasma turbulence in the closed flux surface region, within a diverted geometry. Following what has already been done on the TORPEX device [83-85], the GBS simulation results will also be used to integrate the particle tracer trajectories, in order to study the dynamics of fast ions and impurities in the SOL. Thanks to the code validation methodology we have introduced, it will be possible to assess the accuracy of our simulations at each step in the model improvement.

\section{Acknowledgments}

Many useful discussions with B N Rogers are gratefully acknowledged. We also acknowledge financial support from the Fonds National Suisse de la Recherche Scientifique.

(C) Euratom 2012.

\section{References}

[1] Loarte A et al 2007 Nucl. Fusion 47 S203

[2] Shimada M et al 2007 Nucl. Fusion 47 S1

[3] Stangeby P C 2000 The Plasma Boundary of Magnetic Fusion Devices (Bristol: Institute of Physics Publishing)

[4] Rognlien T D 2005 Plasma Phys. Control. Fusion 47 A283

[5] Braginskii S I 1965 Reviews of Plasma Physics vol 1, ed M A Leontovich (New York: Consultants Bureau) p 205

[6] Mikhailoviskii A B and Tsypin V S 1984 Beitr Plasmaphys. 24335

[7] Dorland W and Hammett G W 1993 Phys. Fluids B 5812

[8] Simakov A N and Catto P J 2003 Phys. Plasmas 104744

[9] Scott B D 2005 Phys. Plasmas 12102307

[10] Zeiler A et al 1997 Phys. Plasmas 42134

[11] Xu X Q et al 2008 Commun. Comput. Phys. 4949

[12] Scott B 2006 Contrib. Plasma Phys. 46714

[13] Naulin V et al 1998 Phys. Rev. Lett. 814148

[14] Garcia O E et al 2004 Phys. Rev. Lett. 92165003

[15] Naulin V and Nielsen A H 2003 SIAM J. Sci. Comput. 25104

[16] Kleva R G and Guzdar P N 2009 Phys. Plasmas 16032305

[17] Kleva R G and Guzdar P N 2007 Phys. Plasmas 14012303

[18] Rogers B N et al 1999 Phys. Rev. Lett. 811941

[19] Scott B 1997 Plasma Phys. Control. Fusion 391635

[20] Sarazin Y and Ghendrih P H 1998 Phys. Plasmas 54214

[21] Bisai N et al 2004 Phys. Plasmas 84018

[22] Myra J R et al 2008 Phys. Plasmas 15032304

[23] Wakatani M and Hasegawa A 1984 Phys. Fluids 27611

[24] Hasegawa A and Wakatani M 1987 Phys. Rev. Lett. 591581

[25] Xu X Q et al 2000 Phys. Plasmas 71951

[26] Xu X Q et al 2002 Nucl. Fusion 4221

[27] Connor J W et al 1998 Phys. Plasmas 52687

[28] Snyder P B et al 2005 Phys. Plasmas 12056115 
[29] Huysmans G T A et al 2009 Plasma Phys. Control. Fusion 51124012

[30] Ricci P et al 2008 Phys. Rev. Lett. 100225002

[31] Ricci P et al 2009 Phys. Plasmas 16055703

[32] Ricci P and Rogers B N 2009 Phys. Plasmas 16062303

[33] Ricci P and Rogers B N 2009 Phys. Plasmas 16092307

[34] Li B et al 2009 Phys. Plasmas 16082510

[35] Ricci P and Rogers B N 2010 Phys. Rev. Lett. 104145001

[36] Rogers B N and Ricci P 2010 Phys. Rev. Lett. 104225002

[37] Ricci P et al 2011 Phys. Plasmas 18032109

[38] Li B et al 2011 Phys. Rev. E 5056406

[39] Furno I et al 2011 Plasma Phys. Control. Fusion 53124016

[40] Gekelman W et al 1991 Rev. Sci. Instrum. 622875

[41] Fasoli A et al 2006 Phys. Plasmas 13055902

[42] Fasoli A et al 2010 Plasma Phys. Control. Fusion 52124020

[43] LaBombard B et al 2005 Nucl. Fusion 451658

[44] Zweben S J et al 2009 Phys. Plasmas 16082505

[45] Cohen R H et al 2007 Nucl. Fusion 47612

[46] Garcia O E et al 2007 Plasma Phys. Control. Fusion 49 B47

[47] D'Ippolito D A et al 2009 Proc. 22nd IAEA Fusion Energy Conf. (Geneva Switzerland 2008) (Vienna: IAEA) paper no IAEA-CN-165/TH/P4-17

[48] Chang C S 2009 J. Phys. Conf. Ser. 180012057

[49] Xu X Q and Dimits A M 2007 49th Annual Meeting of the Division of Plasma Phys. http://meetings.aps.org/link/ BAPS.2007.DPP.CP8.121

[50] Zeiler A 1999 Tokamak Edge Turbulence IPP-Report http://books.google.ch/books?id=HtB1HAAACAAJ

[51] Yu C Q et al 2006 Phys. Plasmas 13042508

[52] Mosetto A et al 2012 Low-frequency linear-mode regimes in the tokamak scrape-off layer Phys. Plasmas at press

[53] Podestà M et al 2006 Plasma Phys. Control. Fusion 481053

[54] Arakawa A 1966 J. Comput. Phys. 1119

[55] Bogacki P and Shampine L F 1989 Appl. Math. Lett. 2321

[56] Amestoy P R et al 2000 Comput. Methods Appl. Mech. Eng. 184501
[57] Schenk O and Gartner K 2004 J. Future Gener. Comput. Syst. 20475

[58] Schenk O and Gartner K 2006 Electr. Trans. Numer. Anal. 23158

[59] Anderson E et al 1999 LAPACK Users' Guide (Philadelphia, PA: Society for Industrial and Applied Mathematics)

[60] Carter T 2006 Phys. Plasmas 13010701

[61] Pace D et al 2008 Phys. Plasmas 15122304

[62] Carter T and Maggs J 2009 Phys. Plasmas 16012304

[63] Horton W et al 2005 Phys. Plasmas 12022303

[64] Perez J et al 2006 Phys. Plasmas 13055701

[65] Berk H L et al 1993 Nucl. Fusion 33263

[66] Wong K L et al 1982 Rev. Sci. Instrum. 53409

[67] Zimmerman E D and Luckhardt S C 1993 J. Fusion Energy 12289

[68] Sharma P K and Bora D 1995 Plasma Phys. Control. Fusion 371003

[69] Gentle K W and Huang H 2008 Plasma Sci. Technol. 10284

[70] Riccardi C et al 1994 Plasma Phys. Control. Fusion 361099

[71] Øynes F J et al 1995 Phys. Rev. Lett. 7581

[72] Rypdal K and Ratynskaia S 2005 Phys. Rev. Lett. 94225002

[73] Riccardi C et al 1997 Phys. Plasmas 43749

[74] Rypdal K and Ratynskaia S 2004 Phys. Plasmas 114623

[75] Perez J C et al 2006 Phys. Plasmas 13032101

[76] Poli F M et al 2006 Phys. Plasmas 13102104

[77] Poli F M et al 2008 Phys. Plasmas 15032104

[78] D'Ippolito D A et al 2011 Phys. Plasmas 18060501

[79] Terry P W et al 2008 Phys. Plasmas 15062503

[80] Greenwald M 2010 Phys. Plasmas 17058101

[81] Lynn A G et al 2009 Rev. Sci. Instrum. 80103501

[82] Loizu J et al 2011 Phys. Rev. E 83016406

[83] Gustafson K et al 2012 Phys. Rev. Lett. 108035006

[84] Gustafson K and Ricci P 2012 Phys. Plasmas 19032304

[85] Gustafson K et al 2012 Phys. Plasmas 19062306 